Research paper

\title{
Effects of asymmetrical damping on a 2 DOF quarter-car model under harmonic excitation
}

\author{
M. Silveira ${ }^{\mathrm{a}, *}$, P. Wahi ${ }^{\mathrm{b}}$, J.C.M. Fernandes ${ }^{\mathrm{a}}$ \\ a Department of Mechanical Engineering, São Paulo State University - UNESP, Bauru, SP, 17033-360, Brazil \\ ${ }^{\mathrm{b}}$ Department of Mechanical Engineering, Indian Institute of Technology - Kanpur, Kanpur-208016, India
}

\section{A R T I C L E I N F O}

\section{Article history:}

Received 20 January 2015

Revised 11 February 2016

Accepted 17 June 2016

Available online 18 June 2016

\section{Keywords:}

Asymmetrical damper

Harmonic balance

Passenger vehicles

Comfort performance

\begin{abstract}
A B S T R A C T
The objective of this work is to study the dynamical behavior of vehicle suspension systems employing asymmetrical viscous damping, with a focus on improving passenger comfort. Previous studies have shown that the use of asymmetrical dampers in these types of systems can be advantageous with regard to comfort of the passengers. The modeling and the behavior of a quarter-car model with asymmetrical viscous damping under harmonic excitation is presented. The response is obtained with an analytical approximation via the method of Harmonic Balance. The choice of the asymmetry ratio diminishes the effects that the uneven road causes on the displacement and acceleration of the sprung mass. Although current systems usually adopt larger damping during the expansion phase, it is shown in this work that, for lower frequencies, smaller damping in this phase results in better comfort.
\end{abstract}

(c) 2016 Elsevier B.V. All rights reserved.

\section{Introduction}

Suspension systems with nonlinear elements have been studied extensively, including nonlinear springs and nonlinear dampers [1-4]. Shekhar et al. showed that the dampers have more influence on the dynamics than the springs [5], and the difference between linear and nonlinear elements is more pronounced for more severe shock loads [6]. These nonlinear elements have been included in active and semi-active suspension systems, in which damping properties vary according to a control algorithm applied, adding or removing energy of the system by means of valves with variable orifice area or magneto-rheological fluids [7-10]. Electromechanical suspension systems have also been studied with great interest as a means of energy recovering system [11,12].

Asymmmetrical dampers are usually designed to provide significantly greater damping force during the extension period compared to the force during the compression period [13]. These different damping coefficients in extension and compression define an asymmetry ratio. The use of an asymmetrical damping induces a shift in the steady state response of the system. The magnitude of this shift depends on the asymmetry ratio and on the excitation frequency [14].

In addition to vertical motion, usually studied with quarter-car models, the pitch displacement and acceleration were shown to be smoother for a half-car model which utilizes asymmetrical dampers [15]. Also, the vertical and angular accelerations reach minimum values at different points in the presence of asymmetrical dampers. Generally, the extension force is

\footnotetext{
* Corresponding author.

E-mail address: m.silveira@feb.unesp.br (M. Silveira).
} 
(a)

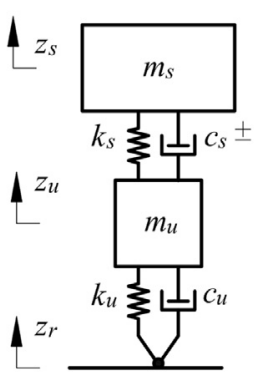

(b)

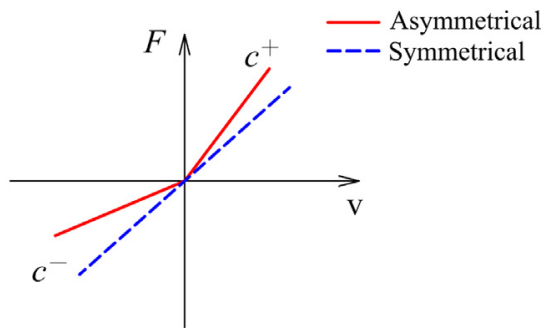

Fig. 1. Two-degree-of-freedom quarter-car model of a suspension system (a) and the generic asymmetrical characteristic of a shock absorber (b).

defined between three to four times the compressive force [16]. However, the variation of this ratio can provide very different behaviours, and has been used in control strategies [17]. The use of more stages of asymmetry has also been studied [18].

It is commonly understood and accepted that the human response to dynamic excitation depends on many mechanical, physical, physiological and psychological parameters [19]. Besides the suspension system per se, the seat of the vehicle is also focus of study to improve comfort in vehicles [20]. The level of comfort for the passengers depends on factors as the frequency of vibration, direction, location and time of exposure to which the passenger is subjected [20]. Apart from causing discomfort, severe vibrations may cause occupational disorders [21].

In order to further explore the use of asymmetrical damping to improve the level of comfort at different frequencies of excitation, in this paper a classical quarter-car model with 2 degrees of freedom is used with harmonic base excitation. The model is presented in Section 2; the response of the system is obtained using an analytical approximation via the method of Harmonic Balance, shown in Section 3; the convergence of the approximate solutions is illustrated in Section 4; the influence of the asymmetry ratio on the response is studied in Section 5; and conclusions are drawn in Section 6.

\section{A 2-DOF quarter-car model with asymmetrical damper}

There are many models suited for the dynamical analysis of vehicles, varying from simpler models to more sophisticated ones. Lower order models with lumped parameters are very common for analysis of the vertical dynamics. Quarter-car models describe exclusively vertical motion of the chassis.

Fig. 1 shows a two-degree-of-freedom shock absorption system. This system comprehends two subsystems connected in series. Each subsystem comprehends a spring and a viscous damper assembled on a structure with mass.

The base of the system represents the road on which the vehicle travels, whose irregularities cause the forces that excite the system. The lower subsystem, comprehended by the mass $m_{u}$, the spring with elastic constant $k_{u}$ and the viscous damper with viscous damping coefficient $c_{u}$, represents the un-sprung mass of the vehicle, e.g. wheels, tyres, brake discs, uprights. These elements have elastic and damper characteristics that, although not being the proper suspension system, contribute to the overall dynamical behaviour of the vehicle. The upper subsystem, comprehended by $m_{s}, k_{s}$, and $c_{s}$, represents the proper suspension system and the sprung mass. The nonlinear absorber is adopted at this subsystem. It is clear that the behaviour of the whole system depends on the contribution of each subsystem, as on the interaction between them.

According to the classical theory of vibrations [22,23], the equation of motion for the system depicted in Fig. 1, regarding vertical displacement relative to the trivial equilibrium positions, can be written as:

$$
\begin{aligned}
m_{u} \ddot{z}_{u}+f_{c u}+f_{k u}-f_{c s}-f_{k s} & =0 \\
m_{s} \ddot{z}_{s}+f_{c s}+f_{k s} & =0
\end{aligned}
$$

The springs are considered linear throughout this study. The spring forces $f_{k u}$ and $f_{k s}$ are proportional to the relative displacement, and are given by:

$$
f_{k u}=k_{u}\left(z_{u}-z_{r}\right) \quad f_{k s}=k_{s}\left(z_{s}-z_{r}\right)
$$

The linear viscous damping force $f_{c u}$ is proportional to the relative velocity, and is given by:

$$
f_{c u}=c_{u}\left(\dot{z}_{u}-\dot{z}_{r}\right)
$$

The nonlinear damping force depends on the relative velocity and its direction. In order to perform the switching of the absorber signal between the viscous damping coefficient at expansion $\left(c_{s}^{+}\right)$and at compression $\left(c_{s}^{-}\right)$, representing its nonlinear characteristic, a Heaviside type function is used as follows:

$$
c_{s}^{ \pm}\left(\dot{z}_{s}-\dot{z}_{u}\right)=\left\{\begin{array}{lll}
c_{s}^{+} & \text {if } & \dot{z}_{s}-\dot{z}_{u} \geq 0 \\
c_{s}^{-} & \text {if } & \dot{z}_{s}-\dot{z}_{u}<0
\end{array}\right.
$$


The base excitation is a result from the irregularities of the road on which the vehicle travels. The base excitation used here is a harmonic function given by

$$
z_{r}(t)=z_{\max } \sin \left(\omega_{r} t+\phi\right)
$$

in which $z_{\max }$ is the amplitude of excitation, $\omega$ is the frequency of excitation and $\phi$ is the phase. The amplitude and frequency of excitation can be tuned to represent different types of roads and different velocities at which the vehicle is travelling. Usually $\phi$ can be set arbitrarily to zero. However, in this case it has to be taken into consideration for the correct match between the responses of the expansion and compression phases.

It is convenient to rewrite Eq. (1) in terms of the relative displacements between unsuspended mass and base displacements, and between suspended mass and unsuspended mass displacements, respectively given by

$$
y_{u}=z_{u}-z_{r} \quad y_{s}=z_{s}-z_{u}
$$

Using equations (6), the equations of motion in terms of relative displacements are given by

$$
\begin{array}{r}
m_{u} \ddot{y}_{u}+m_{u} \ddot{z}_{r}+c_{u} \dot{y}_{u}+k_{u} y_{u}-c_{s}^{ \pm} \dot{y}_{s}-k_{s} y_{s}=0 \\
m_{s} \ddot{y}_{s}-\frac{m_{s}}{m_{u}} c_{u} \dot{y}_{u}-\frac{m_{s}}{m_{u}} k_{u} y_{u}+\left(1+\frac{m_{s}}{m_{u}}\right) c_{s}^{ \pm} \dot{y}_{s}+\left(1+\frac{m_{s}}{m_{u}}\right) k_{s} y_{s}=0
\end{array}
$$

The nondimensional time, mass ratio, stiffness ratio and damping factors for the unsuspended and suspended systems are introduced, respectively, as

$$
\tau=\sqrt{\frac{k_{s}}{m_{s}} t} \quad m=\frac{m_{s}}{m_{u}} \quad k=\frac{k_{u}}{k_{s}} \quad \zeta_{u}=\frac{c_{u}}{2 \sqrt{k_{s} m_{s}}} \quad \zeta_{s}^{ \pm}=\frac{c_{s}^{ \pm}}{2 \sqrt{k_{s} m_{s}}}
$$

Using Eqs. (7) and (8), the nondimensional equations of motion in terms of relative displacement are given by

$$
\begin{array}{r}
\frac{1}{m} y_{u}^{\prime \prime}+\frac{1}{m} z_{r}^{\prime \prime}+2 \zeta_{u} y_{u}^{\prime}+k y_{u}-2 \zeta_{s}^{ \pm} y_{s}^{\prime}-y_{s}=0 \\
y_{s}^{\prime \prime}-2 m \zeta_{u} y_{u}^{\prime}-m k y_{u}+(1+m) 2 \zeta_{s}^{ \pm} y_{s}^{\prime}+(1+m) y_{s}=0
\end{array}
$$

in which a prime denotes differentiation with respect to $\tau$.

\section{Approximate analytical solution using Harmonic Balance}

The system represented by (9) possesses a non-smooth nonlinearity due to the asymmetric damper and can be classified as a piecewise linear system. A semi-analytical exact solution can be obtained by patching together the complete solutions in the two different linear systems with numerical solution of the switching times. However, the algebra becomes involved due to the complicated analytical expressions for the complete coupled system. Since numerical solution of the switching times between the two linear systems is anyway involved in the exact formulation, a closed form semi-analytical approximate solution based on harmonic balance is seeked.

The periodic solution for $y_{s}$ and $y_{u}$ for a given harmonic forcing is approximated as a Fourier series, with the fundamental frequency equal to the excitation frequency. The Fourier coefficients for the approximate solution can be obtained in closed form in terms of the switching times, and numerical solution of the switching times from an algebraic equation is required for approximation involving higher harmonics, although the one-term approximation leads to a closed-form analytical solution. The accuracy of this approximate solution improves with increasing number of harmonics retained in the approximation, as will be shown later. For most practical scenarios, an approximate solution with the first three harmonics is as good as the exact solution. First, the closed form analytical expression obtained for the one-term approximation is presented, followed by a refined solution with more harmonics.

\subsection{Analytical solution using one-term approximation}

For the road profile defined by Eq. (5), the solution for Eq. (9) is seeked in the form

$$
\begin{aligned}
& y_{u}(\tau)=A u_{0}+A u_{1} \cos (\omega \tau)+B u_{1} \sin (\omega \tau) \\
& y_{s}(\tau)=A s_{0}+A s_{1} \cos (\omega \tau)+B s_{1} \sin (\omega \tau)
\end{aligned}
$$

Note that the constant term is necessary for both $y_{u}$ and $y_{s}$ because the asymmetrical non-smooth nonlinearity introduced by the damper appears in the equations for both of them. Substituting this into Eq. (9) results in

$$
\begin{aligned}
C u_{0}+C u_{1} \cos (\omega \tau)+C u_{2} \sin (\omega \tau)+H O H & =0 \\
C s_{0}+C s_{1} \cos (\omega \tau)+C s_{2} \sin (\omega \tau)+H O H & =0
\end{aligned}
$$


in which $\mathrm{HOH}$, standing for higher order harmonics, are a result of the asymmetrical damping term $2 \zeta_{s}^{ \pm} y_{s}^{\prime}$. While writing Eq. (11), the asymmetric damping term $2 \zeta_{s}^{ \pm} y_{s}^{\prime}$, after substituting $y_{s}(\tau)$ from Eq. (10), has been replaced by its Fourier series expansion as

$$
2 \zeta_{s}^{ \pm} y_{s}^{\prime}=c_{0}+\sum_{j=1}^{\infty} c_{2 j-1} \sin (j \omega \tau)+\sum_{j=1}^{\infty} c_{2 j} \cos (j \omega \tau)
$$

The coefficients $c_{0}, c_{2 j-1}$ and $c_{2 j}$ are given by

$$
\begin{aligned}
c_{0} & =\frac{\omega}{2 \pi} \int_{0}^{\frac{2 \pi}{\omega}} 2 \zeta_{s}^{ \pm} \omega\left(-A s_{1} \sin (\omega \tau)+B s_{1} \cos (\omega \tau)\right) d \tau \\
c_{2 j-1} & =\frac{\omega}{\pi} \int_{0}^{\frac{2 \pi}{\omega}} 2 \zeta_{s}^{ \pm} \omega\left(-A s_{1} \sin (\omega \tau)+B s_{1} \cos (\omega \tau)\right) \sin (j \omega \tau) d \tau \\
c_{2 j} & =\frac{\omega}{\pi} \int_{0}^{\frac{2 \pi}{\omega}} 2 \zeta_{s}^{ \pm} \omega\left(-A s_{1} \sin (\omega \tau)+B s_{1} \cos (\omega \tau)\right) \cos (j \omega \tau) d \tau
\end{aligned}
$$

Note that the evaluation of the integrals above requires finding the time instants corresponding to transition from the compression phase $\left(y_{s}^{\prime}<0\right)$, with the damping coefficient as $\zeta_{s}^{-}$, to the expansion phase $\left(y_{s}{ }^{\prime} \geq 0\right)$, with the damping coefficient as $\zeta_{s}^{+}$, and vice versa. The transition times are given accordingly by the two roots of the equation $y_{s}{ }^{\prime}=0$, lying between 0 and $2 \pi / \omega$. Under the one-term approximation for $y_{s}(\tau)$ as given by Eq. (10), the relevant equation is

$$
-A s_{1} \sin (\omega \tau)+B s_{1} \cos (\omega \tau)=0
$$

whose relevant roots are

$$
\tau_{c}=\frac{1}{\omega} \arctan \left(\frac{B s_{1}}{A s_{1}}\right) \text { and } \tau_{c}=\frac{1}{\omega}\left[\arctan \left(\frac{B s_{1}}{A s_{1}}\right) \pm \pi\right]
$$

The choice of the sign in the second root depends on the signs of $A s_{1}$ and $B s_{1}$, so as to ensure that the roots lie in the right interval. For example, if both $A s_{1}$ and $B s_{1}$ are positive, the first root lies in the first quadrant and the second root, with $\mathrm{a}+$ sign, lies in the third quadrant. If both $A s_{1}$ and $B s_{1}$ are negative, the first root is in the third quadrant, while the second root, with a - sign, is in the first quadrant. Note that the above solution for $\tau_{c}$ implies that the damper spends the same amount of time $\pi / \omega$ during the expansion and the compression phases. This is not necessarily true. Hence, there is a need for an approximation involving more harmonics. However, the one-term approximation as above helps in getting a closed form analytical approximation for the periodic solution, and is still of interest in understanding the key qualitative features of the solution. Accordingly, the relevant Fourier coefficients, i.e. $c_{0}, c_{1}$ and $c_{2}$, are evaluated. Without loss of generality, it is assumed that the damper is in the compression phase at the beginning of the cycle. The coefficient $c_{0}$ can be evaluated as

$$
\begin{aligned}
c_{0}= & \frac{\omega^{2}}{\pi}\left[\int_{0}^{\tau_{c}} \zeta^{-}\left(-A s_{1} \sin (\omega \tau)+B s_{1} \cos (\omega \tau)\right) d \tau\right. \\
& +\int_{\tau_{c}}^{\tau_{c}+\frac{\pi}{\omega}} \zeta^{+}\left(-A s_{1} \sin (\omega \tau)+B s_{1} \cos (\omega \tau)\right) d \tau \\
& \left.+\int_{\tau_{c}+\frac{\pi}{\omega}}^{\frac{2 \pi}{\omega}} \zeta^{-}\left(-A s_{1} \sin (\omega \tau)+B s_{1} \cos (\omega \tau)\right) d \tau\right]
\end{aligned}
$$

which reduces to

$$
c_{0}=\frac{2 \omega}{\pi}\left(\zeta^{-}-\zeta^{+}\right)\left[A s_{1} \cos \left(\omega \tau_{c}\right)+B s_{1} \sin \left(\omega \tau_{c}\right)\right] .
$$

In the above, the root for $\tau_{c}$ from Eq. (15) with $\omega \tau_{c}<\pi$ should be used. Similarly, the coefficients $c_{1}$ and $c_{2}$ can be evaluated as

$$
c_{1}=-\omega\left(\zeta^{-}+\zeta^{+}\right) A s_{1} \text { and } c_{2}=\omega\left(\zeta^{-}+\zeta^{+}\right) B s_{1} .
$$

Note again that, under the one-term approximation, the coefficients $c_{1}$ and $c_{2}$ do not depend on the transition time $\tau_{c}$. They also never involve $A s_{0}$, because of the use of the derivative and, therefore, the equations for evaluating the unknown coefficients $A s_{1}, B s_{1}, A u_{1}$ and $B u_{1}$ for the approximation is essentially decoupled from the equations for determining the unknowns $A s_{0}$ and $A u_{0}$.

For Eq. (11) to be valid for arbitrary $\tau$, it is required that the $C s_{i}$ 's as well as $C u_{i}$ 's vanish, yielding the following system of equations

$$
k A u_{0}-\frac{2 \omega}{\pi}\left(\zeta_{s}^{-}-\zeta_{s}^{+}\right)\left[A s_{1} \cos \left(\omega \tau_{c}\right)+B s_{1} \sin \left(\omega \tau_{c}\right)\right]-A s_{0}=0
$$




$$
\begin{aligned}
& \left(k-\frac{\omega^{2}}{m}\right) B u_{1}-B s_{1}-2 \zeta_{u} \omega A u_{1}+\omega\left(\zeta^{-}+\zeta^{+}\right) A s_{1}-\frac{\omega^{2}}{m} A=0 \\
& \left(k-\frac{\omega^{2}}{m}\right) A u_{1}-A s_{1}+2 \zeta_{u} \omega B u_{1}-\omega\left(\zeta^{-}+\zeta^{+}\right) B s_{1}=0 \\
& -m k A u_{0}-\frac{2 \omega}{\pi}\left(\zeta_{s}^{-}-\zeta_{s}^{+}\right)(1+m)\left[A s_{1} \cos \left(\omega \tau_{c}\right)+B s_{1} \sin \left(\omega \tau_{c}\right)\right]+(1+m) A s_{0}=0 \\
& \left(1+m-\omega^{2}\right) B s_{1}-m k B u_{1}-\omega\left(\zeta^{-}+\zeta^{+}\right)(1+m) A s_{1}+2 m \zeta_{u} \omega A u_{1}=0 \\
& \left(1+m-\omega^{2}\right) A s_{1}-m k A u_{1}+\omega\left(\zeta^{-}+\zeta^{+}\right)(1+m) B s_{1}-2 m \zeta_{u} \omega B u_{1}=0
\end{aligned}
$$

Eqs. (20) , (21), (23) and (24) can be solved for $A s_{1}, B s_{1}, A u_{1}$ and $B u_{1}$ as

$$
\begin{aligned}
A s_{1} & =\frac{-m \omega^{3} A\left[k\left(\zeta^{-}+\zeta^{+}\right)\left(-m \omega^{2}-\omega^{2}+m k\right)+4 m \zeta_{u}^{2} \omega^{2}\left(\zeta^{-}+\zeta^{+}\right)+2 \zeta_{u} \omega^{2}\left(1+m-\omega^{2}\right)\right]}{\omega^{8}+a_{6} \omega^{6}+a_{4} \omega^{4}+a_{2} \omega^{2}+k^{2} m^{2}} \\
B s_{1} & =\frac{-m \omega^{2} A\left[k\left(-\omega^{4}+\omega^{2}(k m+m+1)-k m\right)+4 m \zeta_{u}^{2} \omega^{2}\left(\omega^{2}-1\right)+2(1+m) \omega^{4}\left(\zeta^{-}+\zeta^{+}\right)\right]}{\omega^{8}+a_{6} \omega^{6}+a_{4} \omega^{4}+a_{2} \omega^{2}+k^{2} m^{2}} \\
A u_{1} & =\frac{-m \omega^{3} A\left[k m \omega^{2}\left(\zeta^{-}+\zeta^{+}\right)+2 \zeta_{u}\left(\omega^{4}+\omega^{2}\left((1+m)\left(\zeta^{-}+\zeta^{+}\right)^{2}-m-2\right)+m+1\right)\right]}{\omega^{8}+a_{6} \omega^{6}+a_{4} \omega^{4}+a_{2} \omega^{2}+k^{2} m^{2}}
\end{aligned}
$$

and

$$
B u_{1}=\frac{-\omega^{2} A\left[\omega^{6}+b_{4} \omega^{4}+b_{2} \omega^{2}-k m(1+m)\right]}{\omega^{8}+a_{6} \omega^{6}+a_{4} \omega^{4}+a_{2} \omega^{2}+k^{2} m^{2}}
$$

in which

$$
\begin{aligned}
& a_{6}=(1+m)^{2}\left(\zeta^{-}+\zeta^{+}\right)^{2}-2(1+m)+4 m^{2} \zeta_{u}\left(\zeta_{u}+\zeta^{-}+\zeta^{+}\right)-2 m k, \\
& a_{4}=(1+m)^{2}+4 m^{2} \zeta_{u}^{2}\left(\left(\zeta^{-}+\zeta^{+}\right)^{2}-2\right)+k m\left(2(1+m)\left(1-\left(\zeta^{-}+\zeta^{+}\right)^{2}\right)+k m+2\right), \\
& a_{2}=m^{2}\left(k\left(\left(\zeta^{-}+\zeta^{+}\right)^{2} k-2 k-2\right)+4 \zeta_{u}^{2}\right)-2 k m, \\
& b_{4}=m^{2}\left(\zeta^{-}+\zeta^{+}\right)\left(2 \zeta_{u}+\zeta^{-}+\zeta^{+}\right)+m\left(2\left(\zeta^{-}+\zeta^{+}\right)^{2}-k-2\right)+\left(\zeta^{-}+\zeta^{+}\right)^{2}-2,
\end{aligned}
$$

and

$$
b_{2}=m^{2}\left(k-k\left(\zeta^{-}+\zeta^{+}\right)^{2}+1\right)+m\left(2 k-k\left(\zeta^{-}+\zeta^{+}\right)^{2}+2\right)+1 .
$$

From the above solutions for $A u_{1}, B u_{1}, A s_{1}$ and $B s_{1}$, it is difficult to make any judgement about the right sign for $\tau_{c}$ given in Eq. (15) for different ranges of $\omega$. However, one should just remember to use the lower value of $\tau_{c}$ to get the mean shift $A s_{0}$ and $A u_{0}$ from Eqs. (19) and (22). From Eqs. (19) and (22), one can easily find that

$$
A u_{0}=0 \text {. }
$$

The solution for the mean term $A s_{0}$, introduced due to the asymmetrical damper, after duly accounting for the right $\tau_{c}$ is

$$
A s_{0}=\frac{2 m \omega^{3} \sqrt{4 \zeta_{u}^{2} \omega^{2}+k^{2}}\left(\zeta^{-}-\zeta^{+}\right) A}{\pi \sqrt{\omega^{8}+a_{6} \omega^{6}+a_{4} \omega^{4}+a_{2} \omega^{2}+k^{2} m^{2}}}
$$

in which the constants $a_{6}, a_{4}$ and $a_{2}$ are as reported above. Note that $A s_{0}<0$ when $\zeta^{+}>\zeta^{-}$, while $A s_{0}>0$ when $\zeta^{-}<\zeta^{+}$. In general, for vehicle dynamics applications, $\zeta^{+}>\zeta^{-}$, which leads to $A_{0}<0$, referred to as jacking down of the suspension. The final one-term approximation for the absolute displacement of the suspended mass $z_{s}$ under harmonic road profile is given by

$$
\begin{aligned}
z_{s}(\tau) & =z_{r}+y_{u}+y_{s} \\
& =A s_{0}+\left(A s_{1}+A u_{1}\right) \cos (\omega \tau)+\left(A+B s_{1}+B u_{1}\right) \sin (\omega \tau)
\end{aligned}
$$

with the constants $A s_{0}, A s_{1}, A u_{1}, B s_{1}$ and $B u_{1}$ given by Eqs. (25-29). 
With this analytical form for the approximation and an analytical solution for the switching time $\tau_{c}$, the smaller of the two roots in Eq. (15), it is possible to calculate all relevant quantities of interest, such as the energy dissipated by the damper during the compression and the expansion phases. Note that the one-term approximation presented in this subsection implies that the system spends the same time during the compression and the expansion phases, and the energies as calculated from the above analytical approximation are almost the same. To account for the different times spent by the system during the two phases, higher order harmonics are needed, which is presented next.

\subsection{Approximation with higher harmonics}

With the higher harmonics, the solution for Eq. (9) takes the form

$$
y_{s}(\tau)=A s_{0}+\sum_{n=1}^{N}\left[A s_{n} \cos (n \omega \tau)+B s_{n} \sin (n \omega \tau)\right]
$$

and

$$
y_{u}(\tau)=A u_{0}+\sum_{n=1}^{N}\left[A u_{n} \cos (n \omega \tau)+B u_{n} \sin (n \omega \tau)\right],
$$

in which $N$ represents the number of harmonics retained in the approximation. With this approximation, the transition times from the compression to the expansion phase, and vice versa, are given by the first two non-zero roots ( $\tau_{c 1}$, $\tau_{c 2}$, with $\tau_{c 1}<\tau_{c 2}$ ), of the equation

$$
\sum_{n=1}^{N}\left[-n A s_{n} \sin (n \omega \tau)+n B s_{n} \cos (n \omega \tau)\right]=0 .
$$

Again without loss of generality, it is assumed that the cycle starts with the compression phase. Accordingly, the various terms of interest in the Fourier series of the asymmetrical damping term

$$
2 \zeta_{s}^{ \pm} y_{s}^{\prime}=c_{0}+\sum_{j=1}^{N} c_{2 j-1} \sin (j \omega \tau)+\sum_{j=1}^{N} c_{2 j} \cos (j \omega \tau)
$$

can be calculated as

$$
\begin{aligned}
c_{0}= & \frac{\omega^{2}}{\pi}\left[\int_{0}^{\tau_{c 1}} \zeta_{s}^{-} \sum_{n=1}^{N} n\left(-A s_{n} \sin (n \omega \tau)+B s_{n} \cos (n \omega \tau)\right) d \tau\right. \\
+ & \int_{\tau_{c 1}}^{\tau_{c 2}} \zeta_{s}^{+} \sum_{n=1}^{N} n\left(-A s_{n} \sin (n \omega \tau)+B s_{n} \cos (n \omega \tau)\right) d \tau \\
+ & \left.\int_{\tau_{c 2}}^{\frac{2 \pi}{\omega}} \zeta_{s}^{-} \sum_{n=1}^{N} n\left(-A s_{n} \sin (n \omega \tau)+B s_{n} \cos (n \omega \tau)\right) d \tau\right] \\
c_{2 j-1}= & 2 \omega^{2}\left[\int_{0}^{\tau_{c 1}} \zeta_{s}^{-} \sum_{n=1}^{N} n\left(-A s_{n} \sin (n \omega \tau)+B s_{n} \cos (n \omega \tau)\right) \sin (j \omega \tau) d \tau\right. \\
& +\int_{\tau_{c 1}}^{\tau_{c 2}} \zeta_{s}^{+} \sum_{n=1}^{N} n\left(-A s_{n} \sin (n \omega \tau)+B s_{n} \cos (n \omega \tau)\right) \sin (j \omega \tau) d \tau \\
& \left.+\int_{\tau_{c 2}}^{\frac{2 \pi}{\omega}} \zeta_{s}^{-} \sum_{n=1}^{N} n\left(-A s_{n} \sin (n \omega \tau)+B s_{n} \cos (n \omega \tau)\right) \sin (j \omega \tau) d \tau\right]
\end{aligned}
$$

and

$$
\begin{aligned}
c_{2 j}= & \frac{2 \omega^{2}}{\pi}\left[\int_{0}^{\tau_{c 1}} \zeta_{s}^{-} \sum_{n=1}^{N} n\left(-A s_{n} \sin (n \omega \tau)+B s_{n} \cos (n \omega \tau)\right) \cos (j \omega \tau) d \tau\right. \\
& +\int_{\tau_{c 1}}^{\tau_{c 2}} \zeta_{s}^{+} \sum_{n=1}^{N} n\left(-A s_{n} \sin (n \omega \tau)+B s_{n} \cos (n \omega \tau)\right) \cos (j \omega \tau) d \tau \\
& \left.+\int_{\tau_{c 2}}^{\frac{2 \pi}{\omega}} \zeta_{s}^{-} \sum_{n=1}^{N} n\left(-A s_{n} \sin (n \omega \tau)+B s_{n} \cos (n \omega \tau)\right) \cos (j \omega \tau) d \tau\right]
\end{aligned}
$$


using $j=1: N$. Substituting $y_{s}(\tau)$ from Eq. (31), $y_{u}(\tau)$ from Eq. (32) and the Fourier series expansion for the asymmetrical damper from Eq. (34) in Eq. (9), it is possible to write

$$
\begin{gathered}
C u_{0}+\sum_{n=1}^{N}\left[C u_{2 n-1} \cos (n \omega \tau)+C u_{2 n} \sin (n \omega \tau)\right]+H O H=0 \\
C s_{0}+\sum_{n=1}^{N}\left[C s_{2 n-1} \cos (n \omega \tau)+C s_{2 n} \sin (n \omega \tau)\right]+H O H=0
\end{gathered}
$$

in which $\mathrm{HOH}$ stands for the harmonics of order higher than $N$ of the asymmetrical damping term $2 \zeta_{s}^{ \pm} y_{s}{ }^{\prime}$. Again, validity of Eq. (38) for arbitrary $\tau$ is achieved by requiring the various $\mathrm{Cu}_{i}^{\prime} \mathrm{s}$ and $\mathrm{Cs}_{i}^{\prime} \mathrm{s}$ to vanish. This gives $4 \mathrm{~N}+2$ equations, which in conjunction with two equations for $\tau_{c 1}$ and $\tau_{c 2}$, obtained by substituting $\tau_{c}=\tau_{c 1}$ and $\tau_{c}=\tau_{c 2}$ in Eq. (33), gives the complete set of $4 N+4$ equations in the $4 N+4$ unknowns, viz. $\tau_{c 1}, \tau_{c 2}, A s_{0}, A u_{0}, A s_{n}, n=1, \cdots, N, B s_{n}, n=1, \cdots, N, A u_{n}, n=$ $1, \cdots, N$, and $B u_{n}, n=1, \cdots, N$. It is emphasized here again that under the approximation with the higher harmonics, all the Fourier coefficients depend on the transition times, making the coupling stronger, and also causing the entire system of equations to be nonlinear, as opposed to the one-term approximation in which only the coefficient $c_{0}$ dependes on $\tau_{c}$, while the coefficients $c_{1}$ and $c_{2}$ were independent of $\tau_{c}$. However, the equations are linear in the unknown coefficients of the approximation $A s_{0}, A u_{0}, A s_{n}, A u_{n}, B s_{n}$ and $B u_{n}$, and the only source of nonlinearity comes from the unknown transition times $\tau_{c 1}$ and $\tau_{c 2}$. The unknown coefficients $A s_{0}, A u_{0}, A s_{n}, A u_{n}, B s_{n}$ and $B u_{n}$ can be solved in closed form in terms of the forcing parameters $A$ and $\omega$, the system parameters $m, k, \zeta_{u}, \zeta_{s}^{+}$and $\zeta_{s}^{-}$, and the transition times $\tau_{c 1}$ and $\tau_{c 2}$. Substitution of these in Eq. (33) results in a set of coupled equations for the transition times $\tau_{c 1}$ and $\tau_{c 2}$, which have to be solved numerically for each combination of the system and forcing parameters. However, since the nonlinearity has the scaling property (i.e., $\mathrm{f}(\mathrm{ax})=\mathrm{a} f(\mathrm{x})$ ), these equations become independent of the forcing amplitude $A$, and depend only on the forcing frequency $\omega$ and the damping coefficients $\zeta_{u}, \zeta_{s}^{+}$and $\zeta_{s}^{-}$, along with the mass and stiffness ratios, $m$ and $k$.

Once the transitions times $\tau_{c 1}$ and $\tau_{c 2}$ are evaluated numerically, the approximation for the periodic solution of the system is known in closed form, and again all the relevant quantities of interest can be evaluated. The inclusion of the higher harmonics also results in the possibility of evaluating the different times which the system spends in the compression and the expansion phases. The time spent in the expansion phase is $\tau_{c 2}-\tau_{c 1}$, while the time spent during the compression phase is $2 \pi / \omega+\tau_{c 1}-\tau_{c 2}$.

\section{Convergence of approximate analytical solutions}

For illustration of the differences between the solutions from the one-term approximation and the approximation with higher harmonics, the parameters are set to $k=\frac{100}{11}, m=\frac{15}{2}, \zeta_{u}=\frac{\sqrt{66}}{3300}, \zeta_{s}^{+}=\frac{\sqrt{66}}{22}, \zeta_{s}^{-}=\frac{1}{\sqrt{66}}, \omega=\frac{5 \sqrt{66}}{11}$ and $A=1$. All parameters are representative of a medium passenger vehicle (e.g. a sedan carrying four passengers) [3,4,15,24]. The choice of $A$ is not really important due to the scalability property mentioned earlier. The solutions for the transition times are independent of $A$, and the solutions for the unknown coefficients $A_{0}, A_{n}$ and $B_{n}$ can simply be obtained by multiplying the solution for $A=1$ by the desired $A$ value. For this set of parameters, the solution for the one-term approximation is

$$
\begin{aligned}
& y_{s}(\tau)=-0.6753929944-1.160029991 \sin (\omega \tau)+0.1273125741 \cos (\omega \tau) \\
& y_{u}(\tau)=0.05798611519 \sin (\omega \tau)-0.2726469840 \cos (\omega \tau)
\end{aligned}
$$

with the transition time from compression phase to expansion phase as 0.4549754579 , while the transition time from expansion to compression phase is 1.305722770 . The time-period is 1.701494625 , and the system spends 0.8507473122 in both the expansion and the compression phases. The total displacement of the suspended mass is

$$
z_{s}(\tau)=-0.6753929944-0.1020438758 \sin (\omega \tau)-0.1453344099 \cos (\omega \tau) .
$$

For the two-term approximation, the solution is

$$
\begin{aligned}
y_{s}(\tau)= & -0.6781289249-1.155693927 \sin (\omega \tau)+0.1228181574 \cos (\omega \tau) \\
& -0.0842603988 \sin (2 \omega \tau)-0.04093687024 \cos (2 \omega \tau)
\end{aligned}
$$

and

$$
\begin{aligned}
y_{u}(\tau)= & 0.04866710965 \sin (\omega \tau)-0.2630427168 \cos (\omega \tau) \\
& +0.08713668254 \sin (2 \omega \tau)+0.04240858075 \cos (2 \omega \tau)
\end{aligned}
$$

with the transition times $\tau_{c 1}=0.4130503940$ and $\tau_{c 2}=1.332931751$. The system spends 0.9198813570 time units in the expansion phase, which is slightly higher than 0.7816132680 which it spends in the compression phase. The total displacement of the suspended mass is

$$
\begin{aligned}
z_{s}(\tau)= & -0.6781289249-0.1070268174 \sin (\omega \tau)-0.1402245594 \cos (\omega \tau) \\
& +0.00287628372 \sin (2 \omega \tau)+0.00147171051 \cos (2 \omega \tau)
\end{aligned}
$$


Table 1

Switching times with higher harmonics.

\begin{tabular}{lll}
\hline $\mathrm{N}$ & $\tau_{c 1}$ & $\tau_{c 2}$ \\
\hline 1 & 0.4549754579 & 1.305722770 \\
2 & 0.4130503940 & 1.332931751 \\
3 & 0.4003939010 & 1.334942409 \\
4 & 0.4016793221 & 1.337299495 \\
5 & 0.4000764645 & 1.336891773 \\
6 & 0.4004410651 & 1.337329595 \\
7 & 0.4000541553 & 1.337249220 \\
\hline
\end{tabular}

(a)

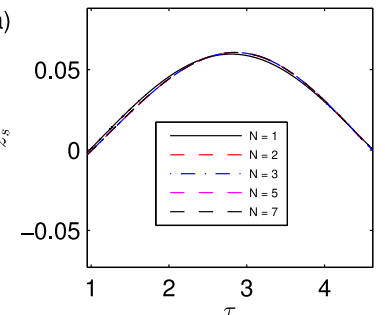

(d)

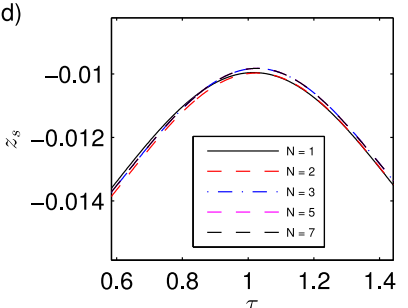

Fig. 2. Displacement of the suspended mass for various numbers of harmonics at $\omega=5$ (a) and $\omega=20$ (b).

Note that the values for $A s_{0}, A u_{0}, A s_{1}, A u_{1}, B s_{1}$ and $B u_{1}$ as obtained from the one-term approximation as well as the twoterm approximation are very close. Also, the magnitude of the coefficients of the second harmonics are at least an order smaller than the coefficients of the terms of the first harmonics, indicating convergence. For this set of parameters, the time spent in the compression and the expansion phases are different, but the difference is not very large and hence, a one-term approximation yielding closed form solutions is a good approximation. For values closer to the resonance, i.e., $\omega=1$, an approximation with higher harmonics is required, because in this regime, the time spent by the system during compression and expansion phases is significantly different. To complete this analysis of the convergence of the approximation, the solution with three harmonics is presented as well, which is

$$
\begin{aligned}
y_{s}(\tau)= & -0.6715757140-1.155735330 \sin (\omega \tau)+0.122548469 \cos (\omega \tau) \\
& -0.09005465407 \sin (2 \omega \tau)-0.04214340498 \cos (2 \omega \tau) \\
& -0.01250716472 \sin (3 \omega \tau)+0.00891232319 \cos (3 \omega \tau)
\end{aligned}
$$

and

$$
\begin{aligned}
y_{u}(\tau)= & 0.0487541786 \sin (\omega \tau)-0.2624645675 \cos (\omega \tau) \\
& +0.09312987609 \sin (2 \omega \tau)+0.04366085332 \cos (2 \omega \tau) \\
& +0.01181099136 \sin (3 \omega \tau)-0.008408776026 \cos (3 \omega \tau)
\end{aligned}
$$

The total displacement of the suspended mass under this approximation is

$$
\begin{aligned}
z_{S}(\tau)= & -0.6715757140-0.1069811513 \sin (\omega \tau)-0.1399160976 \cos (\omega \tau) \\
& +0.00307522202 \sin (2 \omega \tau)+0.00151744834 \cos (2 \omega \tau) \\
& -0.00069617336 \sin (3 \omega \tau)+0.00050354716 \cos (3 \omega \tau)
\end{aligned}
$$

The switching times for the three-term approximation are 0.4003939010 and 1.334942409 , which differ from the ones from the two-term approximation in the second decimal place. To further investigate the convergence aspect, the variation in the transition times with up to $N=7$ is summarised in Table 1 .

From these results, it is possible to safely conclude that an approximation with 4 harmonics is capturing the solution very well for this set of parameters. For values of $\omega$ close to 1, one might require more harmonics to capture the transition times accurately. However, as far as the total displacement of the suspended mass is concerned, even the one-term approximation is very good, as shown in Fig. 2.

\section{Influence of the asymmetry ratio}

Using the parameter values introduced in the previous section, the influence of $\beta$ is studied for different values of $\omega$. Fig. 3 shows the maximum displacement, maximum velocity and maximum acceleration of the suspended mass for varying $\omega$. Three possibilities for values of $\beta$ are used, one representing symmetrical damping $(\beta=1)$, one representing asymmetrical damping with $c^{+}>c^{-}(\beta=3)$, and one representing asymmetrical damping with $c^{+}<c^{-}(\beta=1 / 3)$. Although asym- 

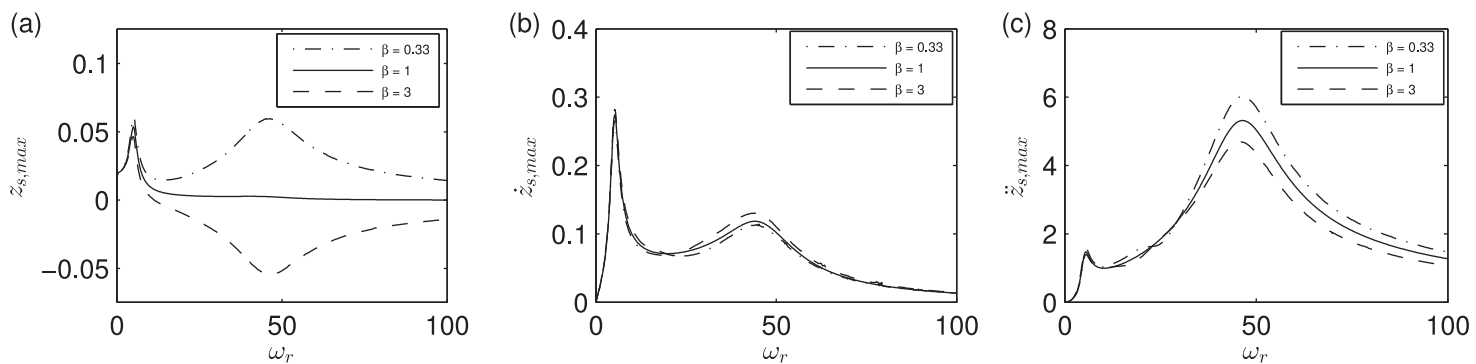

Fig. 3. Maximum displacement (a), maximum velocity (b) and maximum acceleration of the suspended mass for varying excitation frequency with three values of $\beta$.
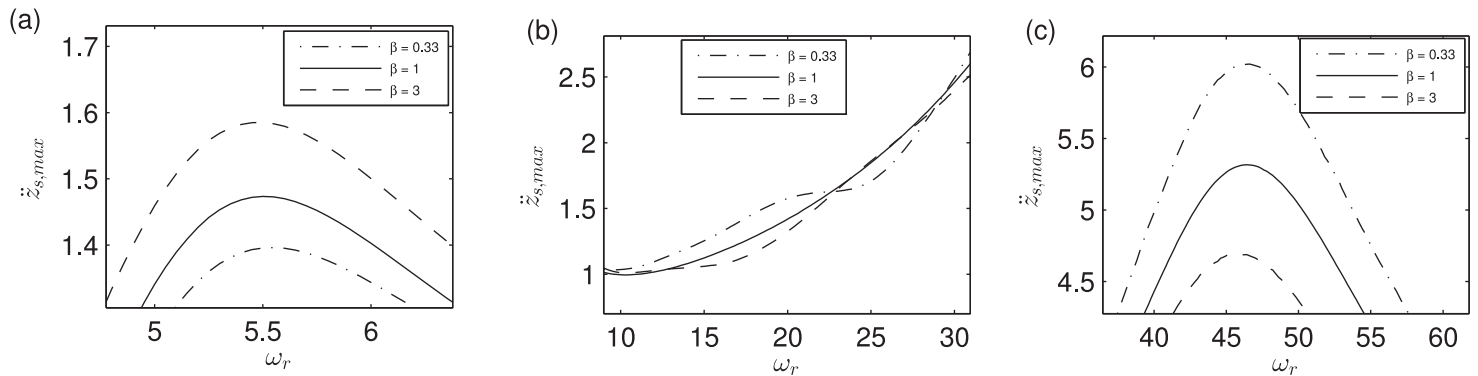

Fig. 4. Maximum acceleration of the suspended mass close to the first resonance peak (a), at intermediate frequencies (b) and close to the second resonance peak (c).
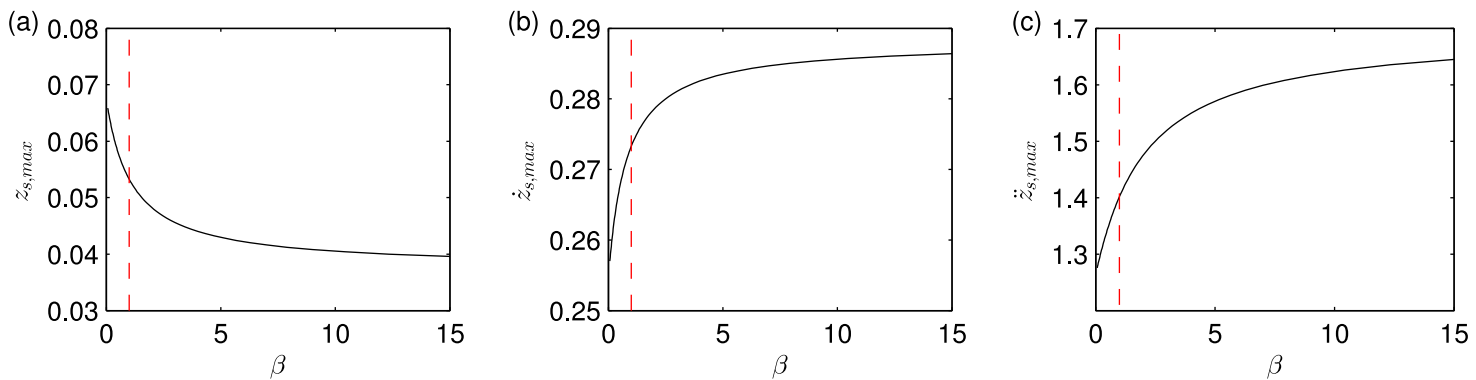

Fig. 5. Maximum displacement (a), maximum velocity (b) and maximum acceleration of the suspended mass for varying asymmetry ratio with $\omega_{r}=5.41$ $\mathrm{rad} / \mathrm{s}$.

metrical dampers in vehicle systems usually have $\beta$ close to 3 [16], it is shown in Fig. 3 that $\beta<1$ results in smaller accelerations for $0<\omega_{r}<20 \mathrm{rad} / \mathrm{s}$.

Comparing the accelerations at the two resonance peaks (Fig. 4), the use of $\beta<1$ presents a reduction of $5.29 \%$ at the first peak, while resulting in an increase of $13.21 \%$ at the second peak when compared to the symmetrical case. The use of $\beta>1$ presents an increase of $7.60 \%$ at the first peak, and a reduction of $13.25 \%$ at the second peak. For both cases of $\beta$, the difference from the symmetrical case diminishes as $\omega_{r}$ increases past $100 \mathrm{rad} / \mathrm{s}$.

Fixing the excitation frequency at the first resonance level, it is possible to see that acceleration values are lower for $\beta<1$ ( Fig. 5). This behaviour reverts at the second resonance level, at which the acceleration values are lower for $\beta>1$ (Fig. 6). Note that this behaviour is dependent on the direction of the acceleration, which is important for comfort perception $[25,26]$. If the absolute value of acceleration is considered, the performance with $\beta<1$ or $\beta>1$ presents advantage over the symmetrical case in the same frequency ranges.

The ratio of energy dissipated during the compression phase and the energy dissipated during the expansion phase is given by

$$
E_{R}=\frac{\int_{0}^{\tau_{c 1}} \zeta_{s}^{-} \dot{x}^{2} \mathrm{~d} t+\int_{\tau_{c 2}}^{2 \pi / \omega} \zeta_{s}^{-} \dot{x}^{2} \mathrm{~d} t}{\int_{\tau_{c 1}}^{\tau_{c 2}} \zeta_{s}^{+} \dot{x}^{2} \mathrm{~d} t}
$$

while the ratio of time spent in the compression phase and time spent in the expansion phase is given by

$$
\tau_{R}=\frac{\tau_{c 1}+2 \pi / \omega-\tau_{c 2}}{\tau_{c 2}-\tau_{c 1}}
$$



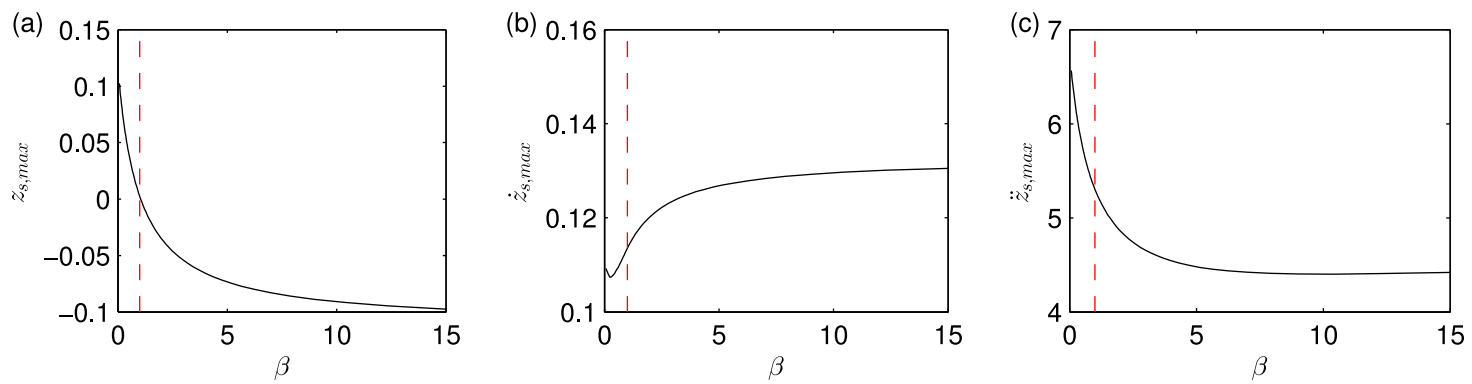

Fig. 6. Maximum displacement (a), maximum velocity (b) and maximum acceleration of the suspended mass for varying asymmetry ratio with $\omega_{r}=44.72$ $\mathrm{rad} / \mathrm{s}$.
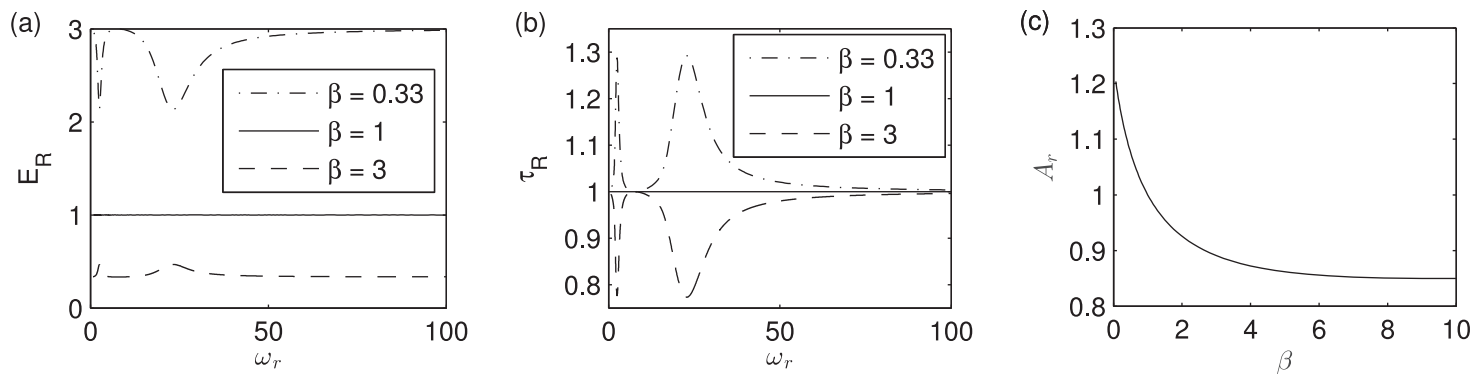

Fig. 7. Energy (a) and time (b) ratios for varying $\omega_{r}$; and ASD ratio for varying $\beta$ (c).

Fig. 7 shows these ratios as function of the excitation frequency. Close to the two resonance peaks, the time spent in the expansion phase is larger with $\beta>1$, while the time spent in the expansion phase is larger with $\beta<1$. However, due to the asymmetrical damping, the magnitude of velocities is higher during the shorter phases, resulting in the opposite behaviour of the energy ratio. Also shown is the asymmetrical to symmetrical ratio of acceleration spectral density $\left(A_{r}\right)$ of acceleration responses for varying $\beta$. Where $A_{r}<1$, the asymmetrical system has a net advantage over the whole frequency range.

\section{Conclusions}

In the analysis of the suspension system using symmetrical and asymmetrical damping with harmonic excitation, it was observed that the asymmetry ratio directly influences the maximum displacement, maximum speed and maximum acceleration of the sprung mass. Regarding the maximum acceleration, the asymmetrical damping proves more advantageous at higher frequencies with $\beta>1$. In the region of lower frequencies, $0<\omega_{r}<20 \mathrm{rad} / \mathrm{s}$, the asymmetrical system with $\beta<1$ proves more advantageous. Athough the average position of the sprung mass varies, the displacement amplitude practically remains. The jacking down effect is caused by the asymmetrical damping characteristics, but the frequency of the harmonic excitation influences the average position of the suspended system.

The difference in acceleration amplitude between the symmetrical and asymmetrical cases is explained by the different dissipation of energy in the system in the expansion and compression phases, and also with the different time spent in each phase, with more time in the phase with lower damping.

Although current systems usually adopt $\beta>1$, it is shown in this work that, for lower frequencies, $\beta<1$ results in better comfort. This observation can be used for devising a control system to tune the value of $\beta$ depending on the road and driving conditions.

\section{Acknowledgements}

The authors thank the Brazilian funding agencies FAPESP (grant \#2014/04768-3), CNPq (grant \#448560/2014-3) and CAPES for the financial support during this project.

\section{References}

[1] Hundal M. Response of shock isolators with linear and quadratic damping. J Sound Vibration 1981;76(2):273-81. doi:10.1016/0022-460X(81)90354-0.

[2] Souza SLT, Caldas IL, Viana RL, Balthazar JM, Brasil RMLRF. Impact dampers for controlling chaos in systems with limited power supply. J Sound Vibration 2005;279:955-67. doi:10.1016/j.jsv.2003.11.068.

[3] Onat C, Kucukdemiral IB, Sivrioglu S, Yuksek I, Cansever G. Lpv gain-scheduling controller design for a non-linear quarter-vehicle active suspension system. Trans Inst Meas Control 2009;31:71-95. doi:10.1177/0142331208090630.

[4] Yagiz N, Hacioglu Y, Taskin YY. Fuzzy sliding-mode control of active suspensions. IEEE Trans Ind Electron 2008;55:3883-90. doi:10.1109/TIE.2008. 924912. 
[5] Shekhar NC, Hatwal H, Mallik AK. Response of non-linear dissipative shock isolators. J Sound Vibration 1998;214:589-603. doi:10.1006/jsvi.1997.1468.

[6] Shekhar NC, Hatwal H, Mallik AK. Performance of non-linear isolators and absorbers to shock excitations. J Sound Vibration 1999;227:293-307. doi:10. 1006/jsvi.1999.2346.

[7] Fischer D, Isermann R. Mechatronic semi-active and active vehicle suspensions. Control Eng Pract 2004;12(11):1353-67. doi:10.1016/j.conengprac.2003. 08.003.

[8] Verros G, Natsiavas S, Papadimitriou C. Design optimization of quarter-car models with passive and semi-active suspensions under random road excitation. J Vibration Control 2005;11(5):581-606. doi:10.1177/1077546305052315.

[9] Tusset AM, Rafikov M, Balthazar JM. An intelligent controller design for magnetorheological damper based on a quarter-car model. J Vibration Control 2009;15:1907-20. doi:10.1177/1077546309102677.

[10] Brown R, Pusey J, Murugan M, Le D. Generalized predictive control algorithm of a simplified ground vehicle suspension system. J Vibration Control 2012. doi: $10.1177 / 1077546312448505$.

[11] Yamapi R. Dynamics of an electromechanical damping device with magnetic coupling. Commun Nonlinear Science Numer Simul 2006;11:907-21. doi:10.1016/j.cnsns.2005.02.001.

[12] Pontes BR Jr, Silveira M, Mazotti AC, Gonçalves PJP, Balthazar JM. Contribution of electrical parameters on the dynamical behaviour of a nonlinear electromagnetic damper. Nonlinear Dyn 2014;79:1957-69. doi:10.1007/s11071-014-1785-3.

[13] Ahmed A, Rakheja S. An equivalent linearization technique for the frequency response analysis of asymmetric dampers. J Sound Vibration 1992;153(3):537-42. doi:10.1016/0022-460X(92)90383-9.

[14] Rajalingham C, Rakheja S. Influence of suspension damper asymmetry on vehicle vibration response to ground excitation. J Sound Vibration 2003;266:1117-29. doi:10.1016/S0022-460X(03)00054-3.

[15] Silveira M, Pontes BR Jr, Balthazar JM. Use of nonlinear asymmetrical shock absorber to improve comfort on passenger vehicles. J Sound Vibration 2014;333:2114-29. doi:10.1016/j.jsv.2013.12.001.

[16] Dixon J. The shock absorber handbook. John Wiley \& Sons; 2007.

[17] Verros G, Natsiavas S, Stepan G. Control and dynamics of quarter-car models with dual-rate damping. J Vibration Control 2000;6:1045-63. doi:10.1177/ 107754630000600706.

[18] Balike K, Rakheja S, Stiharu I. Optimal synthesis of a two-stage asymmetric damper of an automotive suspension considering wheel camber variations. Proc Inst Mech Eng Part D 2011;225(8):1006-22. doi:10.1177/0954407011404294.

[19] Papalukopoulos C, Natsiavas S. Nonlinear biodynamics of passengers coupled with quarter car models. J Sound Vibration 2007;304(1):50-71. doi:10. 1016/j.jsv.2007.01.042.

[20] Boileau PE, Rakheja S. Vibration attenuation performance of suspension seats for off-road forestry vehicles. Int J Ind Ergon 1990;5:275-91. doi:10.1016/ 0169-8141(90)90063-8.

[21] Bouazara M, Richard MJ, Rakheja S. Safety and comfort analysis of a 3-d vehicle model with optimal non-linear active seat suspension. J Terramech 2006;43:97-118. doi:10.1016/j.jterra.2004.10.003.

[22] Dimaragonas AD, Haddad S. Vibration for engineers. London: Prentice-Hall; 1992.

[23] Rao SS. Mechanical vibrations. 3rd ed. Addison-Wesley; 1995.

[24] Akçay H, Türkay S. Influence of tire damping on mixed $\mathcal{H}_{2} / \mathcal{H}_{\infty}$ synthesis of half-car active suspensions. J Sound Vibration 2009;322:15-28. doi:10. 1016/j.jsv.2008.11.007.

[25] DeHart RL. Fundamentals of aerospace medicine. 3rd ed. Lippincott Williams \& Wilkins; 2002.

[26] Nesti A, Barnett-Cowan M, MacNeilage PR, Bülthoff HH. Human sensitivity to vertical self-motion. Exp Brain Res 2014;232:303-14. doi:10.1007/ s00221-013-3741-8 